\title{
Norois
}

Environnement, aménagement, société

$191 \mid 2004 / 2$

Les types de temps

\section{À propos des notions de type de temps et de type de circulation}

\section{François Durand-Dastès}

\section{(2) OpenEdition}

\section{Journals}

Édition électronique

URL : http://journals.openedition.org/norois/1003

DOI : $10.4000 /$ norois. 1003

ISBN : 978-2-7535-1539-0

ISSN : $1760-8546$

Éditeur

Presses universitaires de Rennes

Édition imprimée

Date de publication : 1 mars 2004

Pagination : 29-32

ISBN : 978-2-86847-977-8

ISSN : 0029-182X

\section{Référence électronique}

François Durand-Dastès, «À propos des notions de type de temps et de type de circulation », Norois [En ligne], 191 | 2004/2, mis en ligne le 27 août 2008, consulté le 01 mai 2019. URL : http:// journals.openedition.org/norois/1003 ; DOI : 10.4000/norois.1003

Ce document a été généré automatiquement le 1 mai 2019.

(c) Tous droits réservés 


\title{
À propos des notions de type de temps et de type de circulation ${ }^{1}$
}

\author{
François Durand-Dastès
}

\section{Introduction}

1 La notion de type de temps a été introduite dans les études de climatologie avec le souci :

- de serrer de près la réalité quotidienne ;

- de donner une description concrète des climats, en évitant notamment les inconvénients des statistiques « séparatives »;

- de donner des éléments d'explication du climat, ou encore une « description explicative ».

Ce type de souci était, semble-t-il partagé par tous les participants au colloque de Grenoble. Mais il a semblé à la majorité d'entre eux que des distinctions assez nombreuses devaient être faites, en tenant compte de certains aspects de la réalité et de problèmes d'échelle spatiale et temporelle.

\section{Nécessité d'une distinction entre «type de temps » et «type de circulation »}

3 Une période donnée, pour une portion d'espace donnée, peut être caractérisée par :

- L'ensemble des valeurs prises dans cet espace et pendant cette période par les variables décrivant l'état de l'atmosphère (pluviosité, température, etc.). Cet ensemble de valeurs constitue le «type de temps »; il est décrit qualitativement par des formules du genre " temps chaud et humide », « temps frais et pluvieux », etc.

- L'ensemble des caractères du champ de circulation et de pression, pendant la période donnée, sur la région donnée, ou autour d'elle. C'est le «type de circulation », décrit qualitativement par des formules comme « circulation anticyclonique ", " circulation perturbée de nord-ouest », etc. 
Les deux notions ont souvent été confondues sous le terme général de "type de temps » (sensu lato). La distinction proposée ci-dessus est justifiable par un certain nombre d'arguments. Elle permet de faire référence à la définition classique de Max Sorre «le climat d'un lieu est la série des états de l'atmosphère au-dessus de ce lieu dans leur succession habituelle». Il semble bien que "l'état de l'atmosphère " qui intéresse directement la géographie est bien le «temps qu'il fait » au sens le plus courant du terme. En outre, les travaux qui ne comportent pas la distinction en question présentent un certain nombre d'inconvénients :

- Ils ont un coté abstrait ; en effet, le " type de temps » (sensu lato) est défini essentiellement par un facteur du temps concret, non directement observable. En général, on définit en effet un type de circulation, puis on cherche à donner une idée du temps qu'il fait ce ou ces jourslà, en donnant des tableaux des valeurs moyennes des variables pour la période où règne ce type de circulation. On retrouve alors tous les inconvénients des méthodes séparatives que l'on prétendait éviter, et l'utilisation de la moyenne laisse échapper une part importante de l'information.

- Ils ne sont pas pleinement satisfaisants du point de vue explicatif. En effet, l'explication ne serait vraiment acquise que si le même type de circulation se traduisait toujours par un ensemble relativement homogène d'états de l'atmosphère, ou en d'autres termes, par le même type de temps (sensu stricto). Or il semble que ce ne soit pas toujours le cas, bien au contraire. Aussi devient-il difficile entre autres de rendre compte des aspects concrets du climat, notamment des grandes tendances reflétées par les études statistiques, séparatives ou non (par exemple, tendance à un maximum estival ou hivernal de précipitations, etc.).

Par contre, en utilisant la distinction proposée, on retrouve des possibilités d'explication, suivant le cheminement :

- classification des types de temps (stricto sensu);

- classification des types de circulation ;

- confrontation des deux classements ou des ensembles ainsi constitués.

6 Si les ensembles ne présentent entre eux que des rapports d'inclusion, on arrivera à une image assez simple ; s'il existe des intersections, il faudra expliquer les convergences ainsi mises en évidence.

Deux remarques additionnelles peuvent être faites :

- Le classement des types de temps (stricto sensu) en fonction de plusieurs variables a longtemps été impossible, en raison de l'importance de l'information à traiter. Cela devient maintenant faisable, grâce à l'utilisation des ordinateurs, comme certaines interventions faites à Grenoble l'ont bien montré.

- On a pu dire que le «type de temps » (sensu lato) était en fait un moyen de décrire l'état de l'atmosphère par tous ses caractères, y compris la pression et le vent, donc que la distinction proposée ci-dessus aboutissait à détacher arbitrairement ces deux variables des autres. On peut répondre qu'en fait il s'agit simplement de distinguer les variables qui décrivent une situation en un point, ou sur un espace restreint, de celles qui décrivent un champ, de pression et de mouvement, ce qui est légitime. De toutes façons, il est de bonne logique d'éviter de confondre l'effet et la cause. 


\section{Problèmes d'échelle}

8 La définition des types de circulation et de temps pose des problèmes d'échelle assez délicats :

- Échelle de durée. Temps et circulation sont des états qui reposent sur des observations instantanées. On peut donc se demander pour quelle fraction de la durée ces observations sont valables, quelle est l'échelle temporelle que l'on peut adopter.

- Échelle spatiale. Par définition, le type de circulation repose sur l'étude d'un champ, et implique le recours à une étendue assez vaste, en tout cas à un espace non ponctuel. Quelle est alors la dimension de l'aire à prendre en considération ? Le temps, de son côté, est observé en un point (la station météorologique). Il est donc assez difficile de savoir pour quelle portion d'espace les observations sont valables, autrement dit, quelle est l'extension du type de temps.

On a cherché à répondre à ces questions en proposant un certain nombre de définitions.

\section{Échelles pour les types de temps}

10 C'est le problème de la durée qui semble avoir fait l'objet des interventions les plus nombreuses. Il semble commode de privilégier le cycle diurne, qui est une réalité bien concrète.

On peut alors envisager l'échelle suivante :

- État atmosphérique, météore, phénomène atmosphérique : tous les états dont la durée est inférieure à 24 heures.

- Type de temps : cycle de 24 heures défini par plusieurs variables : total pluviométrique, températures et humidités à certains moments de la journée, amplitudes, etc. (voir par exemple l'utilisation faite de la notion par A. Hufty au Québec).

- Séquence de type de temps : suite ou groupe de journées présentant une certaine homogénéité.

12 Pour les périodes plus longues, ou non homogènes (associant donc plusieurs séquences), on sort en fait du cadre des types de temps, pour entrer dans celui des tendances mensuelles ou saisonnières.

Pour les échelles spatiales, il est plus difficile de proposer un vocabulaire simple. On peut opposer le «temps stationnel » et le temps régional, mais c'est sans doute un peu mince. Il est possible de définir des régions relativement homogènes par rapport au temps (comme les compartiments de M. Vigneau par exemple). MM. Péguy et Mounier ont montré comment on pouvait les délimiter en utilisant des corrélations ; B. Kaiser parle de son coté de «faciès locaux » des types de temps (mais semble en fait faire plutôt référence à des types de circulation).

\section{Échelles pour les types de circulation}

14 Ici, les échelles sont variées en fonction de la réalité, mais aussi du point de vue auquel on se place. En effet, il faut tenir compte à la fois de la dimension du champ de mouvement pris en considération et de la position de la station dans ce champ. Ainsi par exemple, une situation peut être " dépressionnaire ", mais une station peut être en avant ou en arrière 
de la dépression, et il est bon de le préciser. En passant de la description de la circulation " en soi » à la circulation " pour la station », on change souvent d'ordre de grandeur (voir à cet égard une étude non publiée de MM Delannoy et Lecomte).

Il est commode de distinguer les deux aspects du problème et de présenter d'abord une échelle de types de circulation sans tenir compte des positions à l'intérieur du champ. Dans ce cas, il est assez facile de présenter une échelle spatio-temporelle, les systèmes de circulation les plus étendus étant ceux qui ont les plus longues durées. À cet égard, il est aussi commode de procéder d'abord à un classement des types de configuration isobarique, en fonction de leur durée. On peut alors distinguer :

- les figures isobariques semi-permanentes annuelles comme les anticyclones subtropicaux des océans (aucune figure isobarique n'est absolument permanente) ;

- les figures isobariques semi-permanentes saisonnières comme les anticyclones continentaux d'hiver des latitudes moyennes;

- les figures isobariques mobiles à trajectoires préférentielles comme les dépressions des latitudes moyennes, les ondes d'est, etc.

- les figures isobariques exceptionnelles.

En utilisant ces définitions, on peut proposer l'échelle spatio-temporelle ci dessous, qui s'est révélée commode pour décrire, par exemple, la circulation sur l'Inde en été ${ }^{2}$.

- Ordre de grandeur 1 : grands systèmes de circulation, étendus sur plusieurs centaines de milliers à plusieurs millions de $\mathrm{km} 2$, et qui mettent en cause des figures isobariques semipermanentes annuelles ou saisonnières (par exemple, système de la mousson d'été, tourbillon circumpolaire d'ouest, etc.);

- ordre de grandeur 2 : c'est celui du temps synoptique. Du point de vue spatial, il affecte plusieurs dizaines à quelques centaines de milliers de km2. Il met en cause des figures isobariques mobiles à trajectoires préférentielles et c'est à cette échelle qu'apparaissent les déformations et mouvements des figures isobariques annuelles et saisonnières (par exemple, la position du talweg de mousson sur l'Inde, les déplacements des systèmes dépressionnaires d'est dans la même région, etc.) ;

- ordre de grandeur 3 : ici, l'on rencontre des phénomènes étendus sur quelques dizaines à une centaine de milliers de $\mathrm{km} 2$ au maximum. C'est le cas des fronts des latitudes moyennes, des talwegs secondaires et des poussées d'ouest pendant la mousson. L'échelle de durée n'est pas très différente de celle de l'ordre de grandeur 2 ;

- ordre de grandeur 4 : l'unité de mesure est ici la dizaine de $\mathrm{km}$ et le millier de km2, les durées se mesurant en heures. Le phénomène typique est la cellule convective, l'orage, le grain, etc.

Comme nous l'avons dit, il est difficile d'aller très loin en ce qui concerne les différents points de vue, et la position des stations. Notons simplement qu'on est amené à subdiviser tous les types de circulation en introduisant des ordres de grandeur inférieurs à ceux pris en compte au départ. De plus, très souvent, les dimensions se situent plutôt à l'ordre de grandeur 3 ci-dessus.

Notons pour finir qu'il ne faut jamais oublier que tout phénomène d'un certain ordre de grandeur est un ensemble de phénomènes d'ordre de grandeur immédiatement inférieur, et compte parmi ses composantes explicatives un phénomène d'ordre de grandeur immédiatement supérieur. 


\section{NOTES}

1. Note rédigée à la suite des discussions qui ont eu lieu au colloque du CNFG tenu à Grenoble en septembre 1973.

2. La distinction des types et ordres de grandeur de circulation décrite ici a été appliquée au cas de l'Inde dans un article ultérieur : Durand-Dastès (F.), 1979. - « Ordres de grandeur des systèmes de circulation atmosphérique et explication des climats: l'exemple de l'Inde ", L'espace géographique, p. 15-23.

\section{RÉSUMÉS}

Cette courte note insiste d'abord sur la nécessité de distinguer les notions de type de temps et de type de circulation. Le fait de les confondre empêche de poser en termes clairs un problème essentiel pour l'explication des climats, celui du rapport entre le temps qu'il fait et la circulation atmosphérique. Dans un deuxième temps, on propose une grille de classification des types de circulation en fonction des ordres de grandeur du point de vue spatial et temporel.

This short paper at first emphasises the necessity to distinguish between two notions: weather types and atmospheric circulation types. The mixing-up of these different notions prevents from giving a clear treatment to an important matter about the climate explanation: the connection between the observed weather and the atmospheric circulation. Secondly, a framework for the classification of the atmospheric circulation types is proposed, taking spatial and temporal scales into account.

\section{INDEX}

Mots-clés : échelles climatiques, type de circulation, type de temps

Keywords : circulation type, climatological scales, weather type

\section{AUTEUR}

\section{FRANÇOIS DURAND-DASTÈS}

Université Paris 7 